\title{
P210 COMPUTER MODELLING OF FOAM FLOW THROUGH 30 CAPILLARY NETWORK
}

V. ZEMSKIKH and M. KRYLOVA Russian Academy of Sciences, Oil and Gas Research Institute. 19-16 Ul. Mary Ulianovoy, 117331 Moscow. Russia

Abstract. The foam has well-known applications in oil recovery [1]. Applying foam-based methods in IOR requires accurate prediction of it performance which is impossible without an adequate treatment of foam flow. We suggest that a presence of foam in porous media transforms an original capillary size distribution to an effective one. The aim of this paper is to investigate rules of this kind of transformation.

This paper investigates in-situ generated foam flow in water-wetting 3D capillary network presaturated by an aqueous surfactant solution. Foam flow differs from usual water displacement by gas due to the effects of simultaneous co-flowing water and gas in the same system of capillary network channels. This capillaries can be named "foamed". capillaries. We consider gas permeability of that "foamed" capillaries to be less than original one when only gas flows through this system of channels. The decreasing gas permeability of "foamed" capillaries and the rule-based rises of "foamed" capillaries are incorporated in 3-D network simulator of non-steady state unequelibrium displacement of water by gas. It allows consideration of the network as a selfregulating system with respect to gas permeability. The network simulator allows the following functions and parameters to be calculated: saturation on time, permeability on saturation, effective distribution of capillary radii according to sizes such as residual water saturation, effective gas permeability and the structure of foamed clusters. The dependence of gas flowrate through the model on pressure drop to be calculated.

Analysis of the results allows to conclude that the structure of foamed capillaries' clusters and effective radii distribution by sizes depend mainly on pressure drop and reduction permeability of "foamed" capillaries. Fraction of smallest capillaries drastically increases in to the effective distribution of radii by sizes by comparison with original one. The curve shape of effective distribution practically does not change by comparison with its original one for the rest of the range of capillary radius variation.

Introduction. Reduction of gas permeability in the presence of foam can reach several orders without any remarkable influence on permeability to other fluids. Usual simulators account for the presence of foam in porous media by introducing effective gas permeability in the presence of foam, which is less than original one. Another way to understand the influence of foam on gas permeability is to investigate the rules of transformation of original capillary size distribution to effective one. These rules may be more universal than those based on reduction of gas permeability and allow to estimate the gas mobility reduction factor by preliminary calculations. The aim of this work is the computer mathematical simulation of gas flow at the presence of foam through 3-D capillary network.

Network -Description. The sites of a cubic lattice were chosen as the nodes of a capillary network. Cylindrical throats of random radii connected each node with nearest neighbours. Uniform and normal laws of randomisation of throats radii were used. The nodes had no sizes. 
The throats had lengths. The nodes and the throats corresponded to the structure of porous media when the pores sizes were such less compared to the length of throats. We consider nodes as meeting points of throats' ends. Networks with different coordination numbers $\chi$ (the number of throats meeting at a node) were used. Coordination number was chosen as equal to $6,8,10,12$ and 14. The number of network nodes on directions $\mathrm{X}, \mathrm{Y}$ and $\mathrm{Z}$ were specified independently.

The following parameters must be specified before starting the calculations:

- Interfacial tension - $\boldsymbol{\sigma}$ between gas and liquid.

- Contact angles $\theta_{1}$ and $\theta_{2}$.

- Ratio (number of capillaries having contact angle $\left.\theta_{1}\right) /($ number of capillaries having contact angle $\theta_{2}$ ). Both types of capillaries are randomly distributed in the network.

- Gas and liquid viscosities.

- Gas permeability reduction factor of foamed capillaries.

Main assumptions. The fluids are assumed to be incompressible, immiscible and Newtonian. Modified Washburn equation, which takes into account capillary forces, was applied to capillary throats to model meniscus motion [2]. In line of the network structure meniscus passes a node instantaneously. At any moment of time all inner nodes of the network can be divided in two classes: liquid nodes and gas nodes. Under gas nodes we will understand the nodes which have joined throat fully filled with gas. Node will be called «liquid node» if ends of all throats joined contain liquid. We defied active throats as throats which contain both classes of these nodes [3]. We will call throats containing only one class of nodes as non-active. Note that active throat contains only one meniscus while non-active throat either doesn't contain any meniscus at all or contains two menisci. If throat contains two menisci, we consider the permeability of this throat as equal to zero. If we know pressures in every node and position of a menisci at any throat at any moment of time, we consider the state of network as known.

Boundaries and Initial Conditions. Two opposite sides of the network (inlet and outlet) were permeable for fluids and all other sides were impermeable. The pressure at each node of inlet side, $P_{1}$, and the pressure at each node of outlet side, $P_{2}$, were constant during the process of displacement. Initial pressures at all inner nodes were calculated for the network filled with liquid from conservation of flux law in each node and known pressures at inlet and outlet nodes by means of the system of equations:

$$
\sum_{j=1}^{\chi} \frac{r_{j}^{2}\left(P_{j}-P_{0 i}\right)}{L_{j}}=0
$$

where: $r_{j}$ - radius of «j» -throat; $L_{j}$ - length of $\left.\langle\mathrm{j}\rangle\right\rangle$-throat; $P_{0 i}$ - pressure at given central $\langle\mathrm{i}\rangle-$ node; $P_{j}$ - pressure at nearest neighbouring «j»-node; $\mathrm{i}=1,2, \ldots, \mathrm{N} ; \mathrm{N}$ - the number of inner nodes. Network was filled with liquid before the starting of the displacement simulation. At the initial moment inlet nodes were specified as gas nodes.

Menisci Motion Calculations. As mentioned above meniscus can move only in an active throat. Dimensionless modified Washburn equation, describing the motion of meniscus through every active throat, takes the following form:

$$
\frac{d \xi_{f_{j}}}{d \tau}=\frac{R_{j}^{2}\left(P_{j}-P_{0 i}+A_{j} P_{j}^{c}\right)}{C_{j} \xi_{f_{j}}+D_{j}\left(L_{j}-\xi_{f_{j}}\right)}
$$


where: $\xi_{f_{i}}(t)$ - coordinate of meniscus position at «j»-throat; $C_{j}=\mu_{g} / \mu_{l}, D_{j}=1$ or $C_{j}=1, D_{j}=\mu_{g} / \mu_{l}$ - parameters which depend on the location of liquid and gas at «j»-throat with respect to direction of flow; $A_{j}=-1$ or $A_{j}=1$ - parameter which depends on the direction of flow at (j)»-throat; $P_{j}^{\mathrm{c}}$ - capillary pressure at 《j»-throat. Initial condition for equation (2) at any time step is $\xi_{f}(0)=\xi_{f_{0}}$, where $0 \leq \xi_{f_{0}} \leq L_{j}$. The meniscus can move within any throat in any direction according to relation between the values of pressures at the ends of the throat and the value of capillary pressure. The time interval which takes motion to the end of throat was calculated by the means of equation (2) for each active throat. The minimal among these time intervals was taken as the next time step of the code. Co-ordinates of all menisci at active throats were recalculated for next time step. Thus, at any time step the one of throats was fully filled with liquid or gas. If gas comes to the liquid node through this fully gas filled throat menisci instantly arise to the ends of all the others throats joined with this node. If after that any throat contains two menisci, this throat becomes non-active with zero permeability.

Foam Generation. The direction of meniscus' motion may be changed in the process of the displacement. In this case liquid can come to a gas node (the liquid across gas path). We considered this situation as a very favourable for foam generation. Simultaneously gas and liquid flows can arise in the throats from which gas leaves the node. Permeability of this kind of lamellae throats declines due to the reduction of cross-section area of throat occupied by gas or due to arising [4]. We will call these capillaries as «foamed» capillaries. Gas permeability reduction factor, GPRF, accounting for reduction of permeability of foamed capillaries, is the parameter of the problem. This parameter is equal to the ratio of permeability of foamed throat to original one and may be a number from interval $(0 ; 1]$.

Pressure Field Calculation. We consider the pressures in all nodes a constant until the distribution of menisci to throats doesn't change. If meniscus comes to a liquid node, and the distribution of menisci to the throats is changed; pressure field has to be recalculated to take into account the new distribution of menisci (and, as consequence, capillary pressure too) to the throats.

Under these assumptions subproblem of pressure field calculation is reduced to numerical solving of second order elliptical equation (analogous to Laplace's equation) with variable coefficients on the grid corresponding to lattice of the network:

$$
\sum_{j=1}^{\chi} f M_{j} \frac{R_{j}^{2}\left(P_{j}-P_{0 i}+A_{j} P_{j}^{c}\right)}{C_{j} \xi_{j}+D_{j}\left(L_{j}-\xi_{j}\right)}=0
$$

Here $f M_{j}$ is equal to 0,1 or value of GPRF for impermeable, usual and foamed throats correspondingly. This equations have been derived from the conditions of fluxes conservation in the nodes. All simbols correspond to the one defined above. Time stabilisation method has been used to solve this problem.

Results and Discussion. As follows from mentioned above, the described algorithm simulates unsteady and nonequilibrium displacement of liquid by gas in 3-D network with self regulating permeability to gas. At the last stage of the calculation, time step of code drastically increases on several orders. It means that any meniscus motion to an end of a throat takes very long time compared to usual one. We consider that in this case the equilibrium was reached between action of capillary forces and pressure field in the network. Systematic runs of the code for wide range 
of the problem parameters show that permeability of the network at this last stage depends on both pressure drop and permeability reduction factor (GPRF).

Fig. 1 shows an example of the gas flux dependencies on pressure drop for different values of GPRF. We observe that these dependencies have non-linear character. It means that the structure of clusters, formed by foamed capillaries, depends on both GPRF and pressure drop.

Fig. 2 shows the dependence of foamed capillaries' fraction on GPRF at the fixed pressure drop. We can see that the number of foamed capillaries increases with decreasing GPRF. (Decreasing of GPRF means decreasing of foamed throats permeability to gas). Note that values of foamed capillary fraction for rather significant degree of gas permeability reduction are above the percolation threshold for 3-D cubic lattice. It means that foamed capillaries can form an infinite cluster. In this case we can talk about infinite cluster of capillaries with simultaneous gas and liquid flow. This is in contradiction with the usual main assumption of two phase flow theory about each liquid occupying its own system of channels in porous media. In our case we may find in the network three infinite clusters: occupied by liquid, gas and foam (gas dispersed in liquid).

Foamed capillary with reduced permeability may be treated like capillary with reduced radius compared to original one. From this point of view we have ability to consider some effective distribution of throats to size and compare it with original one. Fig. 3 and 4 show these dependencies of radii density distribution by sizes on radii for normal and uniform law of distribution correspondingly. We can obsrve that in both cases distributions have two modes. Curves 1 at both figures correspond to original distribution. They have one mode. Second mode, having small radii and high density, arises in effective distributions. This mode is formed by foamed capillaries. As mentioned above fraction of capillaries forming this mode is around thirty percent of all capillaries. The remaining part of capillaries is distributed practically according to original laws. This is an unexpected result because, seeming, foam has tendency to occupy capillaries of largest radii.

\section{Conclusions.}

1. Pressure drop and degree of gas permeability reduction have influence on the formation of foamed capillary clusters in the network.

2. Foamed capillaries can constitute infinite cluster in the network which can be treated as a system of connected capillaries with simultaneous gas and liquid flow.

3. The effective distribution of radii by sizes has two modes. First, there is a mode with smallest radii, which is formed by foamed capillaries. Second mode is the distribution of the remaining part of capillaries has the same shape that original one:

\section{References}

1. "Foams: Fundamentals and Applications in the Petroleum Industry", edited by L.L. Schramm, Adventures in Chemistry Series 242, American Chemical Society, Washington, DC, 1994.

2. Washburn, E.W.: «The Dynamic of Capillary Flow», Phys. Rev., 1921, vol. 17, 273-283.

3. Entov I.M., Feldman A.Y., Chen-Sin A., «Modeling of displacement process in Porous Media», Moscow, Isvestia AN SSSR, Programmirovanie, 1975, N3, pp. 67-74 (in Russian).

4. Zemskikh V., Krylova M. «Physical and Mathematical Modeling of Foam Flow Through Porous Median, presented at I-st International Workshop of Reservoir Application of Foam, 2-5 April, 1993, Stavanger, Norway. 


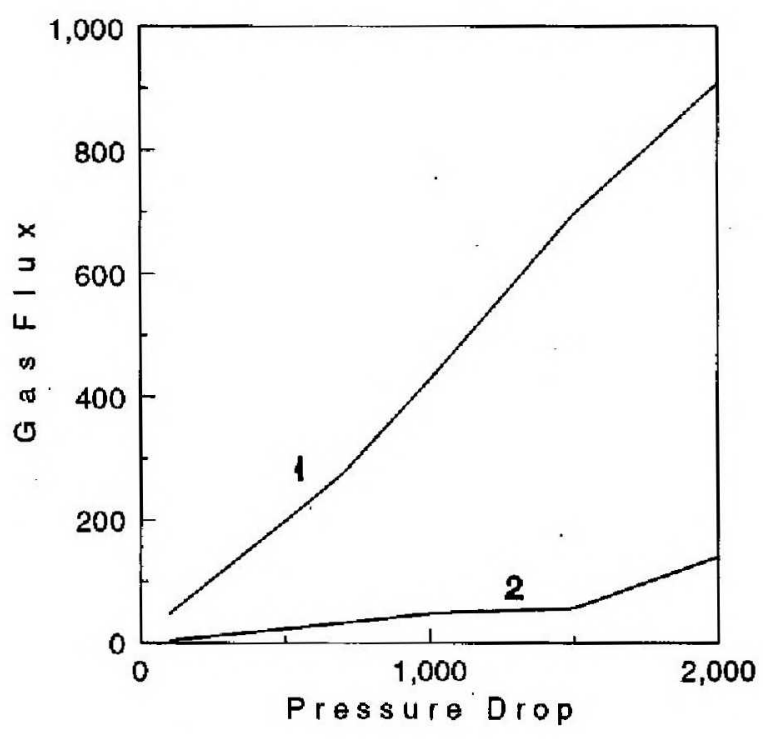

Fig. 1. Dependence of gas flux on pressures drop.

Network dimension $5 \times 5 \times 5 ; \chi=6 ; \sigma=70 ; \theta_{1}=0.11, \theta_{2}=0.22$; gas/liquid viscosity ratio - 0.01 1 - GPRF=0.01; 2 - GPRF=0.001.

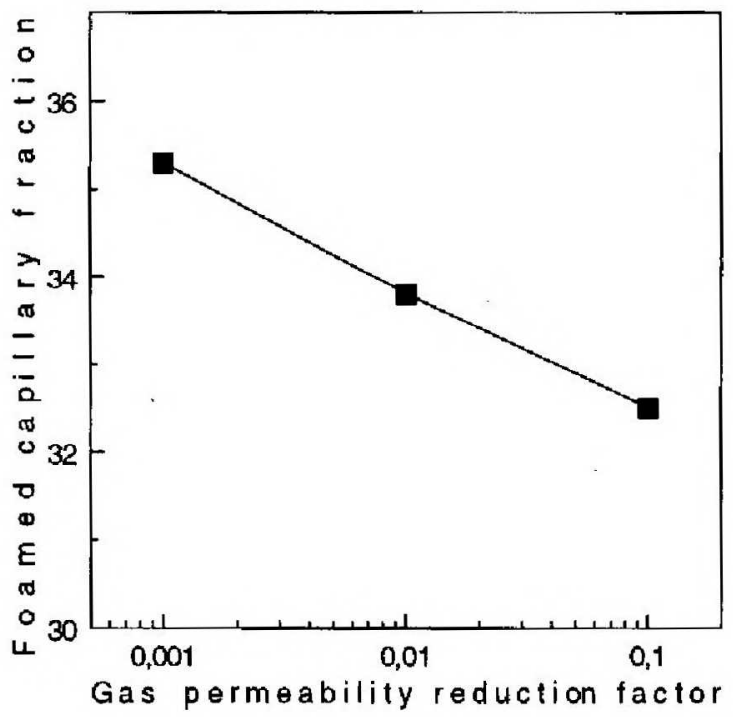

Fig. 2. Dependence of foamed capillary fraction on gas permeability reduction factor.

Network dimension $5 \times 5 \times 5 ; \chi=6 ; \sigma=70 ; \theta_{1}=0.11, \theta_{2}=0.22$; gas/liquid viscosity ratio -0.01 . 


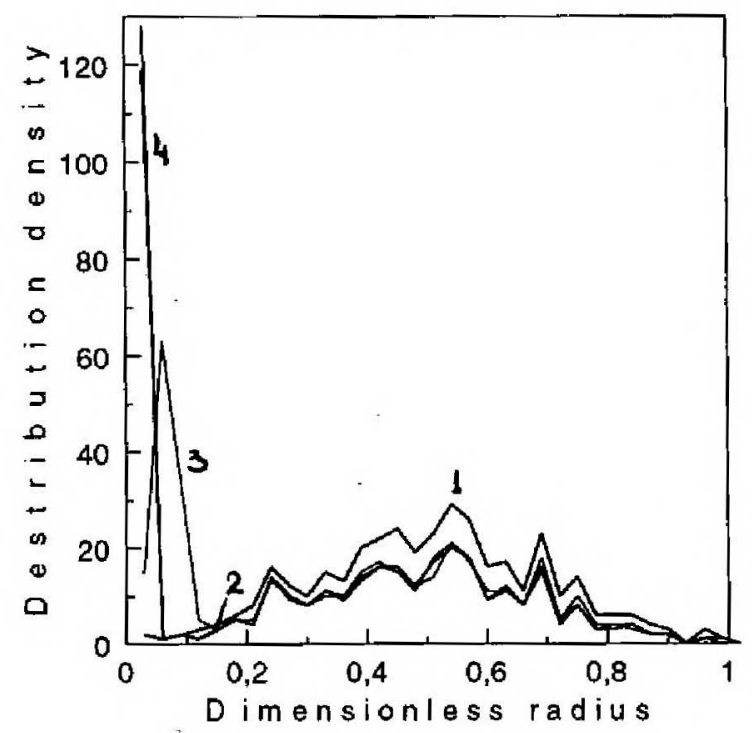

Fig. 3. Dependence of density distribution on radius size.

Network dimension $5 \times 5 \times 5 ; \chi=6 ; \sigma=70 ; \theta_{1}=0.11, \theta_{2}=0.22$; normal distribution (middle radius - 0.5 , dispersion -0.2 ); ratio gas viscosity/liquid viscosity - $0.01 ; \mathrm{P}_{1}=300 ; \mathrm{P}_{2}=200$; $1-\mathrm{GPRF}=1 ; 2-\mathrm{GPRF}=0.1 ; 3-\mathrm{GPRF}=0.01 ; 4-\mathrm{GPRF}=0.001$.

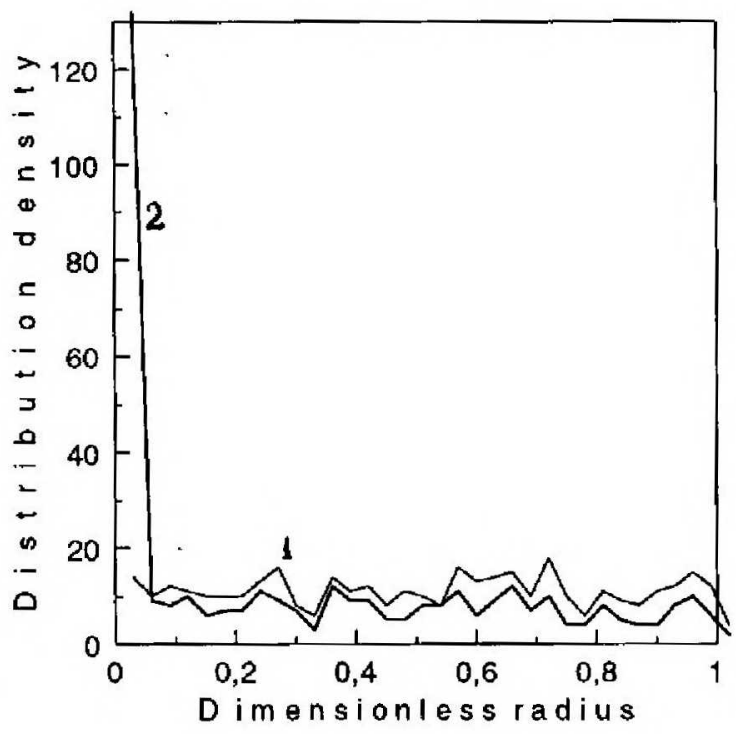

Fig. 4. Dependence of density distribution on radius size.

Network dimension $5 \times 5 \times 5 ; \chi=6 ; \sigma=70 ; \theta_{1}=0.11, \theta_{2}=0.22$; uniform distribution; ratio gas viscosity/liquid viscosity - 0.01 ; $\mathrm{P}_{1}=300 ; \mathrm{P}_{2}=200$; $1-\mathrm{GPRF}=1 ; 2-\mathrm{GPRF}=0.01$. 